\title{
Balkanologie
}

Balkanologie Revue d'études pluridisciplinaires

Vol. $15 n^{\circ} 1 \mid 2020$

Mémoires performatives : faire des passés et des présents

\section{Enis Sulstarova, Arratisje nga Lindja : Orientalizmi shqiptar nga Naimi te Kadareja [Fuir l'Orient : l'orientalisme albanais de Naim Frashëri à Ismail Kadare]}

Tirana, Pika pa sipërfaqe, 2016

Lola Sinoimeri

\section{OpenEdition}

Édition électronique

URL : https://journals.openedition.org/balkanologie/2552

DOI : 10.4000/balkanologie. 2552

ISSN : 1965-0582

Éditeur

Association française d'études sur les Balkans (Afebalk)

Référence électronique

Lola Sinoimeri, «Enis Sulstarova, Arratisje nga Lindja : Orientalizmi shqiptar nga Naimi te Kadareja [Fuir I'Orient : I'orientalisme albanais de Naim Frashëri à Ismail Kadare] », Balkanologie [En ligne], Vol. 15 nº $^{\circ}$ 1 | 2020, mis en ligne le 01 juin 2020, consulté le 05 août 2021. URL : http://journals.openedition.org/ balkanologie/2552 ; DOI : https://doi.org/10.4000/balkanologie.2552

Ce document a été généré automatiquement le 5 août 2021.

(c) Tous droits réservés 


\section{Enis Sulstarova, Arratisje nga Lindja : Orientalizmi shqiptar nga Naimi te Kadareja [Fuir l'Orient: l'orientalisme albanais de Naim Frashëri à Ismail Kadare]}

Tirana, Pika pa sipërfaqe, 2016

Lola Sinoimeri

\section{RÉFÉRENCE}

Sulstarova, Enis, 2016, Arratisje nga Lindja: Orientalizmi shqiptar nga Naimi te Kadareja [Fuir l'Orient : l'orientalisme albanais de Naim Frashëri à Ismail Kadare], Tirana, Pika pa sipërfaqe, 333 pages, ISBN 978-9928-4136-7-3

1 Enis Sulstarova est chercheur en science politique et sociologie à l'Université de Tirana. La majeure partie de ses travaux (thèse de doctorat, articles et ouvrages publiés) porte sur les nationalismes albanais ainsi que sur ce qu'il nomme «l'orientalisme albanais » à différentes époques, du mouvement dit de «renaissance nationale» à la fin du $\mathrm{XIX}^{\mathrm{e}}$ siècle jusqu'à l'époque contemporaine. Arratisje nga lindja [Fuir l'Orient] constitue l'aboutissement de ces recherches sur « l'orientalisme albanais » et sur le rôle de celuici dans la construction d'une identité nationale. L'ouvrage, paru initialement en 2006 en langue albanaise, a connu trois rééditions, dont la plus récente est de $2019^{1}$. L'auteur explique, dans l'avant-propos de l'édition de 2016, qu'il a mis à jour le chapitre sur la période contemporaine mais qu'il est également revenu sur le premier chapitre afin d'y inclure des données issues de travaux réalisés entre la première et la troisième édition. L'ouvrage a connu une bonne réception dans les milieux de la recherche universitaire 
albanophone mais a eu très peu d'écho à l'international, notamment à cause de l'absence de traduction'.

Dans le premier chapitre, l'auteur se positionne par rapport au concept d'orientalisme, tel qu'il a été élaboré par Edward Saïd à partir des années 1970, et identifie les spécificités de ce qu'il appelle l'«orientalisme albanais». Sulstarova part des différentes dimensions de l'orientalisme définies par Saï. L'orientalisme peut d'abord être compris comme un domaine d'études (ensemble des connaissances sur les sociétés dites orientales) produit par un certain nombre d'institutions d'enseignement et de recherche. Mais il peut aussi être défini plus largement comme "un style de pensée fondé sur la distinction ontologique et épistémologique entre 'l'Orient' et (...) 'l'Occident' ${ }^{\prime}$ structuré par des couples d'opposés (immobilité contre progrès, superstition contre rationalité, infériorité contre supériorité). Enfin, une troisième acception décrit l'orientalisme comme «un style occidental de domination, de restructuration et d'autorité sur l'Orient ${ }^{5} »$, notamment dans le contexte historique de la colonisation. Si ces trois dimensions sont indissociables l'une de l'autre chez Saïd, Enis Sulstarova n'en retient pour sa méthodologie que la deuxième, discursive. Dans son premier chapitre, il souligne en effet que «l'orientalisme albanais » n'est pas le résultat d'un passé colonial de l'Albanie, ni d'une tradition universitaire de recherche sur "l'Orient». Ainsi, selon Sulstarova, les « orientalistes albanais» ne sont ni des savants ni des administrateurs coloniaux, « mais ce sont pour la plupart des écrivains, poètes, journalistes, faiseurs d'opinion et autres intellectuels qui considèrent comme acquise la distinction ontologique entre Occident et Orient et qui utilisent cette distinction comme point de départ de créations artistiques, de descriptions, d'analyses sociologiques ou de discours politiques avec pour visée principale la (re)construction de l'identité moderne albanaise ${ }^{6} »$. Sulstarova fait un parallèle entre la production des discours orientalistes en Albanie et d'autres pays non occidentaux comme la Russie ou la Turquie ${ }^{7}$. On y constate l'appropriation d'un discours orientaliste pour penser les trajectoires de leurs propres sociétés : l'Occident devient alors l'horizon civilisationnel à atteindre, tandis que l'Orient est à fuir. L'orientalisme dans ces pays est, selon l'auteur, un orientalisme « interne »: il « agit à l'intérieur de l'État ", dans le sens où il a pour finalité la désorientalisation des éléments et sujets considérés comme orientaux. Ainsi, l'orientalisme albanais est interne quand il définit certaines populations de la société albanaise comme orientales (par exemple, les populations musulmanes ou les populations non blanches, comme les communautés roms ou égyptiennes). Mais, selon Sulstarova, l'orientalisme albanais est également externe dans la mesure où le discours orientaliste albanais oppose l'identité albanaise à l'orient, c'est-à-dire aux sociétés du Moyen-Orient. L'auteur s'appuie également sur les analyses de Maria Todorova ${ }^{8}$ sur le «balkanisme » et rappelle le cadre théorique des débats à propos de la distinction, ou non, entre orientalisme et balkanisme. Sans se situer clairement d'un côté ou de l'autre de ce débat théorique, il affirme que Todorova elle-même reconnaît l'existence d'un certain orientalisme dans la manière dont les peuples balkaniques rivalisent entre eux d'européanité pour se rapprocher de l'Occident - ce que l'autrice Milica Bakić-Hayden nomme « nesting orientalism » [orientalisme emboîté]".

3 La suite de l'ouvrage, à partir du chapitre 2, se déroule de manière chronologique, de la fin du xIx ${ }^{e}$ siècle jusqu'à nos jours. Le choix d'embrasser plus d'un siècle d'histoire et la construction chronologique de l'argumentation permettent à l'auteur de mettre l'accent sur les continuités qui caractérisent le discours orientaliste en Albanie et sur la 
capacité d'adaptation de ce discours aux changements et aux ruptures politiques. Selon Sulstarova, la sémantique orientaliste albanaise a toujours joué un rôle central dans la production des discours nationalistes et plus généralement identitaires.

4 Les chapitres 2, 3 et 4 éclairent le rôle joué par les discours orientalistes dans la construction d'un État et d'une identité nationale albanaise depuis la formation d'un mouvement nationaliste albanais à partir de la fin du xixe siècle jusqu'au début de la période communiste. Sulstarova montre comment le chemin jusqu'à l'indépendance vis-à-vis de l'Empire ottoman en 1912 se fait en opposition avec les "Turcs ${ }^{10}$ ", symbole de «despotisme » et d' " arriération ${ }^{11}$ ». Les textes littéraires analysés par Sulstarova représentent alors à la fois des modèles à suivre d'Albanais occidentaux et des repoussoirs orientaux. Ainsi, à la fin du $\mathrm{xIX}^{\mathrm{e}}$ siècle, le poète Naïm Frashëri fait de Skanderbeg, seigneur albanais $\mathrm{du} \mathrm{xv}^{\mathrm{e}}$ siècle, à la fois le héros national albanais par excellence et le défenseur de la civilisation occidentale contre le pouvoir ottoman ${ }^{12}$. Dans les années 1930, la publication par l'écrivain Mitrush Kuteli du récit Vjersha e Xheladin Beut [L'Automne du bey Xheladin] ${ }^{13}$ contribue à cristalliser un imaginaire négatif autour du bey ${ }^{14}$ albanais, fortement inspiré de la figure historique d'Ali Pacha de Janina, gouverneur ottoman de l'Épire au début du XIX siècle. On y retrouve tout l'imaginaire du despote oriental, dépravé et cruel, figure qui a fait son apparition dans la pensée politique et philosophique européenne de l'époque des Lumières ${ }^{15}$.

5 Après l'indépendance de l'Albanie en 1912, et notamment durant la période d'entredeux-guerres, l'idée qui sous-tend les écrits des intellectuels est celle de l'européanité des Albanais.es, qui, après cinq cents ans d'« obscurantisme » ottoman, seraient prêts à se tourner à nouveau vers l'Europe en suivant la voie du progrès et du modernisme tracée par les penseurs occidentaux des Lumières et en s'inspirant des modèles des États occidentaux. Enis Sulstarova s'appuie notamment sur les théories politiques de deux auteurs albanais. Selon les écrits de Krist Maloki, admirateur des régimes fascistes italiens et allemands, «l'Orient » constituerait « l'ennemi invisible » à l'intérieur même de "l'âme albanaise », qu'il s'agirait de purifier après l'occupation ottomane. Branko Merxhani, s'inspirant de l'idéologie nationaliste turque et notamment de l'auteur Ziya Gökalp, est quant à lui l'initiateur du mouvement « néo-albanianiste » (neoshqiptarizëm). Selon lui, il ne s'agit pas de copier simplement l'Occident mais d'adapter les institutions occidentales à «l'âme albanaise » qu'il faut débarrasser de toute trace d'« orientalité ». Bien que leurs idéologies politiques soient opposées, on retrouve chez ces deux penseurs nationalistes l'idée que les cinq cents ans d'Empire ottoman ne constituent qu'une parenthèse passagère dans l'histoire des Albanais.es et n'ont en rien altéré la pureté de «l'âme albanaise ».

6 Durant la période communiste, analysée dans le chapitre 5, le mépris pour les régimes d'Europe occidentale ne remet pas en cause l'identité nationale albanaise telle qu'elle s'est construite jusque-là. Selon les discours politiques d'Enver Hoxha lui-même, l'Albanie se destine au contraire à sauver l'Europe occidentale de la menace capitaliste, de la même manière qu'elle a constitué un rempart contre les Ottomans au $\mathrm{Xv}^{\mathrm{e}}$ siècle : le régime qui se met en place se réclame d'une continuité forte avec les intellectuels de la fin du XIX ${ }^{\mathrm{e}}$ siècle et mobilise le même imaginaire nationaliste de modèles occidentaux et repoussoirs orientaux.

7 C'est l'analyse des œuvres d'Ismail Kadare qui occupe le plus de place dans le chapitre dédié à la période communiste, mais également dans le suivant qui traite de la période post-communiste. Sulstarova anticipe la critique qu'on pourrait lui faire de se 
concentrer sur l'œuvre d'un seul auteur sur cette période en insistant sur trois points. Premièrement, l'œuvre de Kadare serait selon lui, malgré les débats, représentative du réalisme socialiste puisqu'elle promeut la figure de "l'homme nouveau» et en particulier de "l'Albanais nouveau». Deuxièmement, comme Kadare est l'écrivain albanais le plus lu en Albanie comme à l'international, il jouerait un rôle considérable dans la culture albanaise et dans la représentation d'une identité albanaise qui s'inscrit dans la continuité des périodes précédentes. Enfin, les œuvres de Kadare seraient éminemment politiques, dans le sens où elles reflèteraient les idéologies dominantes du régime, ainsi que ses changements d'orientations politiques. Ainsi, son œuvre illustre parfaitement la manière dont l'orientalisme albanais s'adapte à ces changements ainsi qu'à la chute du régime : au moment de la rupture avec l'URSS puis avec la Chine, les révisionnismes soviétiques puis chinois sont diabolisés comme des menaces orientales qui remettraient en cause l'européanité originelle de l'Albanie ${ }^{16}$. Dans les œuvres de la période post-communiste, c'est Enver Hoxha lui-même qui est plus ou moins explicitement présenté comme un agent dont le but serait de "ré-orientaliser » la société albanaise ${ }^{17}$. Si l'orientalisme de Kadare est avant tout politique durant la période communiste, il va de soi que cet orientalisme nourrit un imaginaire orientaliste de l'identité albanaise et de la figure de «l'Albanais nouveau ».

Le chapitre 6, qui porte sur la période post-communiste, ne se limite pas à l'analyse des romans de Kadare, mais se concentre également sur des discours et essais politiques d'autres intellectuels albanais, comme le chercheur Aurel Plasari ${ }^{18}$ ou le journaliste Piro Misha $^{19}$, qui aspirent avant tout à l'intégration de l'Albanie dans l'Union européenne (UE). L'orientalisme prend la forme d'une islamophobie et d'un racisme interne et externe ; interne contre les populations musulmanes et non blanches en Albanie, mais aussi au Kosovo et en Macédoine; externe contre les pays plus à l'est, plus " orientaux ", c'est-à-dire considérés comme moins européens. Ainsi, la potentielle intégration de la Turquie à l'UE poserait problème à l'Albanie, qui ne parvient toujours pas à accéder au rang de candidate officielle et donc à faire reconnaître son européanité aux pays d'Europe occidentale. L'auteur s'attarde également sur la querelle qui a lieu entre l'écrivain kosovar Rexhep Qosja ${ }^{20}$ et Ismail Kadare ${ }^{21}$ : pour le premier, l'identité albanaise se situe au croisement de deux civilisations (bien que critique de la vision monolithique de l'identité albanaise défendue par Kadare, Rexhep Qosja ne dépasse pas la dualité Orient/Occident), tandis que Kadare défend une identité exclusivement occidentale et donc catholique et blanche - et ce, au mépris de la majorité confessionnelle musulmane en Albanie.

9 Sulstarova clôt son ouvrage sur une conclusion pour le moins étonnante qui pointe le «retard» de la recherche albanaise par rapport aux pays occidentaux, où le développement des études postcoloniales aurait permis le « dépassement » de ce type de discours orientalistes. L'auteur rappelle certes que cet orientalisme est bien " emprunté » aux anciennes puissances coloniales mais il me semble qu'il aurait été intéressant de souligner davantage l'actualité de cet "emprunt», qui n'est pas à reléguer à une période coloniale désormais révolue. Plus qu'un emprunt d'ailleurs, il s'agit bien d'une intériorisation et il serait important de penser la notion d'« orientalisme intérieur» comme indissociable d'un "orientalisme intériorisé » propre aux pays des Balkans : que l'orientalisme albanais soit interne ou externe, il est le fruit de ce que Sulstarova lui-même appelle un "complexe d'infériorité22" par rapport à l'Occident. Derrière cette expression, je crois qu'il faut comprendre en réalité 
un mécanisme de domination culturelle, historique et géopolitique des pays d'Europe occidentale sur les Balkans, sur lequel l'auteur insiste peu.

Or, insister sur les mécanismes de transfert non seulement entre l'Europe occidentale et les Balkans (par exemple, beaucoup de chercheurs cités par Sulstarova ont été formés ou ont exercé dans les universités d'Europe de l'Ouest, comme Krist Maloki, Branko Merxhani, Aurel Plasari entre autres, mais l'auteur ne l'évoque pas), mais également à l'intérieur des Balkans et à l'intérieur de l'Albanie, en utilisant le concept de Bakić-Hayden de "nesting orientalism » [orientalisme emboîté] comme un vrai outil d'analyse, aurait permis de comprendre mieux encore les contextes d'apparition de la sémantique orientaliste dans les pays non occidentaux ainsi que les parallèles que l'on peut faire entre les différents " orientalismes non occidentaux ». En réalité, malgré les convictions de l'auteur, on peut lui reprocher un nationalisme méthodologique dans le choix des auteurs. Ce choix empêche de voir ces mécanismes de transferts à l'extérieur et à l'intérieur de la société albanaise.

11 D'ailleurs, alors que Sulstarova insiste tant sur l'idée d'un « orientalisme intérieur » qui stigmatise certaines populations albanaises, il parait très étonnant d'évincer à ce point les contre-discours possibles : le corpus choisi par l'auteur est en effet exclusivement masculin et semble constitué d'auteurs blancs, en grande partie non musulmans hormis quelques contre-discours analysés pour souligner l'hégémonie du discours orientaliste albanais, comme celui de Qosja. De fait, beaucoup de questions se posent après la lecture : par quels mécanismes de transfert ces discours orientalistes sont-ils devenus dominants en Albanie, un pays à majorité confessionnelle musulmane ? Où sont les contre-discours, les voix subalternes, féminines, roms, musulmanes? Quel est le rôle du genre et des sexualités dans la création de l'altérité « orientale » à l'intérieur et à l'extérieur de l'Albanie ? Défaire ou du moins interroger l'homogénéité trop forte de ce corpus sur plus d'un siècle d'« orientalisme albanais » permettrait de comprendre encore mieux sa spécificité par rapport aux discours orientalistes émanant d'Europe de l'Ouest ainsi que les liens possibles avec eux.

\section{NOTES}

1. L'auteur y a modifié quelques passages par rapport à l'édition de 2016. Mais, comme la présente note de lecture a été écrite pendant la période de confinement de mars à mai 2020 en France, je n'ai pas pu accéder matériellement à la dernière édition. Je me suis donc appuyée sur l'édition de 2016.

2. Voir notamment le compte rendu suivant: PULA Besnik, «Review of Arratisje nga lindja: orientalizmi shqiptar nga Naimi te Kadare by Enis Sulstarova », Albanian Journal of Politics, vol. II, $\mathrm{n}^{\circ} 1$, 2006, p. 72-81.

3. SAIID Edward W., L'Orientalisme : l'Orient créé par l'Occident, C. Malamoud et C. Wauthier (trad.), Paris, Éditions du Seuil, 1997, p. 31.

4. Ibid., p. 31 .

5. Ibid., p. 32.

6. SULSTAROVA, op. cit., p. 37. 
7. Sur les discours orientalistes en Russie et en Turquie, il cite notamment les travaux suivants : ANDREEVA Elena, Russia and Iran in the Great Game: Travelogues and Orientalism, Londres, Routledge, 2007 ; ZEYDANLIOĞLU Welat, "The White Turkish Man's Burden: Orientalism, Kemalism and the Kurds in Turkey", dans Guido Rings, Anne Ife (dir.), Neo-colonial Mentalities in Contemporary Europe? Language and Discourse in the Construction of Identities, Newcastle upon Tyne, Cambridge Scholars Publishing, 2008.

8. Todorova Maria, Imaginaire des Balkans, R. Bouyssou (trad.), Paris, Éd. de l'École des hautes études en sciences sociales, 2011.

9. BAKIĆ-HAYDEN Milica, "Nesting Orientalisms: The Case of Former Yugoslavia ", Slavic Review, vol. 54, n 4, 1995, p. 917-931.

10. Précisons que la désignation «Turcs" est en soi ambiguë car elle peut renvoyer dans de nombreuses langues des Balkans à la fois aux Ottomans, aux Turcophones, aux Turcs de Turquie, aux Musulmans (parfois albanais). L'auteur ne précise cependant pas ce que le mot "Turc" contient d'ambigu dans un contexte balkanique, sans doute car il écrit lui-même en langue albanaise. Dans le contexte de la période dite de "renaissance nationale ", il s'agit pour les nationalistes albanais de se distancier de l'Empire ottoman en usant d'une sémantique orientaliste empruntée aux penseurs des Lumières, comme par exemple ce que l'on peut lire à propos de la Turquie dans l'Encyclopédie de Diderot et d'Alembert.

11. Voir: CLAYER Nathalie, Aux origines du nationalisme albanais: la naissance d'une nation majoritairement musulmane en Europe, Paris, Éd. Karthala et CERI, 2018.

12. FRASHËRI Naim, Histori e Skënderbeut [Histoire de Skanderbeg], Tirana, Naim Frashëri,1967.

13. KUTELI Mitrush, Vepra letrare, vëll 1. [๕uvres littéraires, vol. 1], Tirana, Naim Frashëri, 1989.

14. Il s'agit d'un titre qui désigne sous l'Empire ottoman un « chef de clan ».

15. Voir : THOMSON Ann, «L'Empire ottoman, symbole du despotisme oriental ? ", dans Isabelle Gadoin, Marie-Élise Palmier-Chatelain (dir.), Rêver d'Orient, connaître l'Orient : visions de l'Orient dans l'art et la littérature britanniques, Lyon, ENS Éditions, 2014, p. 177-196.

16. KADARE Ismail, Le grand hiver, Jusuf Vrioni (trad.), Paris, Gallimard, 1990.

17. KADARE Ismail, Les adieux du mal, Jusuf Vrioni (trad.), Paris, Stock, 1996.

18. PLASARI Aurel, Vija e Teodosit Rishfaqet: nga do t'ia mbajnë shqiptarët? [Le retour de la frontière de Théodose : où se placeront les Albanais ?], Tirana, S.l., 1992.

19. MISHA Piro, Duke kërkuar rrënjët ose... kthimi i shqiptarëve në histori [À la recherche des racines... ou le retour des Albanais dans l'histoire], Tirana, Toena, 1997.

20. QOSJA Rexhep, Ideologjia e shpërbërjes: trajtesë mbi idetë çintegruese në shoqërinë e sotme shqiptare [L'Idéologie de la désintégration: commentaire sur les idées de désintégration dans la société albanaise contemporaine], Tirana, Toena, 2006 ; Realiteti i shpërfillur : vështrim kritik mbi pikëpamjet e Kadaresë për identitetin shqiptar [Négliger la réalité : une critique des points de vue de Kadare sur l'identité albanaise], Tirana, Toena, 2006.

21. KADARE Ismail, Identiteti evropian $i$ shqiptarëve [L'Identité européenne des Albanais], Tirana, Onufri, 2006

22. SULSTAROVA, op. cit., p. 309.

INDEX

Index géographique : Albanie 


\section{AUTEURS}

LOLA SINOIMERI

Université Paris 8 Vincennes Saint-Denis 